\title{
Against Extrinsic Dispositions
}

\begin{abstract}
McKitrick (2003) proposes that an object has a disposition if and only if there are a manifestation, the circumstances of the manifestation, a counterfactual true of the object, and an overtly dispositional locution referring to the disposition. A disposition is extrinsic if and only if an object has it, but a perfect duplicate of the object might not have it. I present an alternative definition that an object has a disposition if and only if a counterfactual is true of the object that under a certain condition, it would interact with another object in a certain manner. There are three reasons for thinking that my definition is better than her definition. 1 . Ockham's razor favors my definition over McKitrick's definition. My definition is consistent, while her definition is not, with Lewis's and her definitions of intrinsic and extrinsic properties. 3. My definition goes well, while her definition does not, with our intuition that an object has a disposition even in a possible world where there is nothing but that object.
\end{abstract}

\section{Keywords}

Counterfactual Interaction, Extrinsic Disposition, Fragility, Intrinsic Disposition, Toxicity

Park, Seungbae (2017). "Against Extrinsic Dispositions," Review of Contemporary Philosophy 16: 92-103.

https://www.addletonacademicpublishers.com/contents-rcp/944-volume-16-2017/3092against-extrinsic-dispositions

Acknowledgement: This paper benefited from the metaphysics of science course taught by Dr. Christian Wüthrich and Dr. Tomasz Bigaj at University of California - San Diego in 2015.

Seungbae Park

Ulsan National Institute of Science and Technology

Republic of Korea

nature@unist.ac.kr

\section{Introductions}

Are there extrinsic dispositions? Philosophers have different answers to this question. Mark Johnston (1992: 234), George Molnar (1999: 3), and David Lewis (1997: 148) say no, claiming that all dispositions are intrinsic. Jennifer McKitrick (2003) says yes, contending that some dispositions are extrinsic. She defines 'extrinsic disposition' and then provides six examples that she claims fit her definition. Her definition and examples are original and intriguing, constituting a strong case for the existence of extrinsic dispositions.

This paper aims to show, however, that McKitrick's definition and six examples are all problematic. I proceed as follows. In Section 2, I clarify the concepts of intrinsic and extrinsic properties, which are crucial for understanding McKitrick's argument for the existence of extrinsic dispositions. In Section 3, I present my definition and McKitrick's definition of a disposition, and then bring up an important difference between the two definitions. In Section 4, I criticize her six examples, arguing that Ockham's razor favors my definition over her definition of extrinsic disposition, and that my definition goes well, while her definition does not, with Lewis's and her definitions of intrinsic and extrinsic properties. In Section 5, I argue that my definition meshes well, while her definition does not, with our intuition that an object 
has a disposition even in a possible world where there is nothing but that object. In the end, it will become clear that McKitrick has failed to show that there are extrinsic dispositions.

\section{Intrinsic vs. Extrinsic}

We first need to be clear about the distinction between intrinsic and extrinsic properties because a clear understanding of the distinction will enable us to assess adequately McKitrick's definition and examples of extrinsic dispositions. Lewis and McKitrick state the distinction as follows:

We distinguish intrinsic properties, which things have in virtue of the way they themselves are, from extrinsic properties, which they have in virtue of their relations or lack of relations to other things. (Lewis, 1986: 61)

Intuitively, a property is intrinsic if anything that has it has it regardless of what is going on outside of itself. Perfect duplicates necessarily share intrinsic properties. Extrinsic properties, by contrast, are simply those that are not intrinsic. If a property is extrinsic, it is possible that a thing's having that property depends on what is going on outside of the thing. Perfect duplicates can differ with respect to their extrinsic properties. (McKitrick, 2003: 158)

For example, I have the property of being male and the property of being a brother. The former is an intrinsic property, while the latter is an extrinsic property. I have the property of being male by virtue of the way I am. I would retain the property, even if my brother ceased to exist. My having the property does not depend on what is going on outside of me. By contrast, I have the property of being a brother in virtue of my relation to my brother. I would lose that property, if he ceased to exist. Thus, my having the property depends on what is going on outside of me.

Two things are noteworthy. First, Lewis and McKitrick share the same conception of intrinsic and extrinsic properties. Second, an extrinsic property does not make any intrinsic difference to an object. Suppose that $I$ at some time $t_{1}$ and $I$ at another time $t_{2}$ are perfect duplicates. The duplicates do not differ in terms of intrinsic properties. They would, however, differ in terms of extrinsic properties, if $I$ had a brother at $t_{1}$, but not at $t_{2}$. Keep these two points in mind. They will turn out to be useful in my critical discussion of McKitrick's case for the existence of extrinsic dispositions below.

\section{Rivaling Definitions}

\subsection{My Definition}

The concept of a disposition can be illustrated by the following two paradigm examples. First, a piece of glass has the dispositional property of being fragile (McKitrick, 2003: 156). Second, cyanide has the disposition to kill human beings (Tomasz Bigaj, Personal Communication). From these two examples, we can infer a general condition under which we can attribute a disposition to an object. I propose that an object has a disposition if and only if there is a counterfactual true of the object that under a certain condition, an event would occur that brings about changes to it and to another object, i.e., that under a certain condition, the two objects would interact with each other in a certain manner. Such an interaction might be called a counterfactual interaction. The preceding two paradigm examples of disposition have relevant counterfactual interactions. If a hammer moves in a certain direction with a certain force, it would interact with the glass in a certain way. Specifically, it would acquire some negligible scratches, and the glass would break. If a human being takes cyanide, the cyanide would interact with the human being in a certain manner. Specifically, the cyanide would spread inside the human body, and the human body would stop functioning. These two 
examples indicate that a counterfactual interaction is required for us to attribute a disposition to an object.

I use these two examples because there is an interesting difference between them. When the glass interacts with the hammer, the glass undergoes a conspicuous change, and we attribute the disposition to the object that undergoes the conspicuous change. When the cyanide interacts with a human being, the cyanide produces a conspicuous change to the human being, and we attribute the disposition to the object that produces the conspicuous change. Thus, we can attribute a disposition to an object, regardless of whether it undergoes or generates a conspicuous change. It is not the case that only an object that undergoes a salient change, or only an object that generates a salient change, is a legitimate target of our disposition attribution.

A disposition is causally efficacious. Consider again that if the glass is struck by the hammer, the glass would break. What makes the glass break? The hammer's smashing and the disposition of the glass jointly generate the event. The hammer's striking alone could not produce the event. After all, if the glass were not fragile, it would not break even if it was struck by the hammer. Consider also that if someone took the cyanide, he would die. $\mathrm{He}$ would die because the cyanide has the disposition to kill a human being. Keep in mind that a disposition of an object is causally potent in that it makes a difference to the way the object interacts with another object.

Are the dispositions of the glass to break and of the cyanide to kill human beings intrinsic or extrinsic properties? The disposition to break is an intrinsic property, given that it is a property that the glass has in virtue of the way it is. The glass would retain that property, even if the hammer were destroyed. Besides, the disposition is reducible to the atomic structure of the glass. Its atomic structure is a property the glass has in virtue of the way it is, and it is independent of the relations that the glass bears to other objects, such as a hammer. Similar reasoning applies to the disposition of cyanide to kill a human being. It appears, therefore, that all dispositions are intrinsic properties of their bearers.

McKitrick, however, argues that some dispositions are extrinsic. Let me turn to her case for the existence of extrinsic dispositions in the following section.

\subsection{McKitrick's Definition}

McKitrick's six examples of extrinsic dispositions are not the kind of properties that we can observe with our naked eyes, as will become clear in this section. How does she argue for the existence of these unobservable properties? Her method can be made clear by comparing it with scientists' method to argue for the existence of theoretical entities, such as electrons and black holes.

Scientists use inference to the best explanation (IBE) to justify their belief that theoretical entities are real. For example, the kinetic theory of heat competed with the caloric theory of heat. Scientists chose the kinetic theory on the grounds that it better explained heat

phenomena. So we are justified in believing that molecules, which are postulated by the kinetic theory, are real. A similar story explains why we are justified in believing that black holes, which are posited by the general theory of relativity, are real.

Like scientists, metaphysicians can use IBE to establish their belief in unobservable properties. They can argue, for example, that glass has the unobservable property of being fragile because fragility of glass best explains why it breaks when struck by a hammer. McKitrick, however, does not use IBE to argue for the existence of extrinsic dispositions. She uses a totally different method, so far as I can tell. Her method is to offer a definition and examples that she claims fit the definition. Thus, we need to engage ourselves with her definition and examples to evaluate her case for the existence of extrinsic dispositions. 
How does McKitrick define 'disposition'? She lists four marks of dispositionality and contends that if a property has enough of them, it is probably a disposition:

In general, if a property exhibits enough marks of dispositionality, there is good reason to think it is a disposition. Consider a property P. Does P have a characteristic manifestation? Are there circumstances that would typically bring about the occurrence of this manifestation? Is there a certain counterfactual that is typically true of things that possess P? Is P named by an overtly dispositional locution? If so, then $P$ is probably a disposition. (McKitrick, 2003: 158)

Fragility exhibits all the four marks. The glass's breaking is the manifestation of fragility. The hammer's striking the glass is the circumstances that would bring about the manifestation. The counterfactual is true of the glass that if the glass were struck by a hammer, it would break. The overtly dispositional locution 'the disposition to break when struck by a hammer' refers to the property of being fragile. It follows that fragility is a disposition. Similar reasoning applies to the disposition of cyanide to kill human beings. Both fragility and toxicity fit McKitrick's definition of disposition.

Suppose that we have identified a particular disposition of an object by using this method. How do we tell whether the disposition is intrinsic or extrinsic? According to McKitrick, we only need to perform a thought experiment. A disposition of an object is extrinsic if and only if the object loses the disposition when its environment changes in a certain way (McKitrick, 2003: 159). In other words, a disposition is extrinsic, if and only if perfect duplicates situated in different environments might not share it, i.e., if and only if an object has the disposition, but a perfect duplicate of the object might not have it because the duplicate has been placed in a different environment.

Let me bring up an important difference between my definition and McKitrick's definition of disposition. My definition requires an interaction between objects, whereas McKitrick's definition does not. Recall that according to her definition, there should be a manifestation and the circumstances of the manifestation. These circumstances are not necessarily contiguous to an object. So there might not be a causal relationship between the circumstances and the object. This small difference has a grave philosophical consequence for her examples of visibility and recognizability, as will become clear below.

\section{Examples}

\subsection{Key, Weight, and Water}

McKitrick approvingly cites Sydney Shoemaker's (1984: 221) example of a key, claiming that a "key has a disposition to unlock a certain door" (2003: 159). Under the circumstances in which the key is turned, its disposition to open a door is exhibited, i.e., the manifestation occurs. The counterfactual is true of the key that if the key is "placed in the lock and turned, the door would open" (McKitrick, 2003: 259). The overtly dispositional locution "the disposition to open the door' refers to the disposition of the key to open the door.

How do we know that the key's disposition to open the door is extrinsic? She appeals to Shoemaker's contention that the key could lose the disposition when its environment changes:

As Shoemaker points out, the key can lose this property if someone changes its environment, more specifically, if someone changes the lock on the door. An object at some time $t_{1}$ can be a perfect duplicate of itself at a later time $t_{2}$. So, consider a key at $t_{1}$ that is able to open door $\mathrm{x}$. Between $t_{1}$ and $t_{2}$, the lock on door $x$ is changed. Now, a perfect duplicate of the key at $t_{1}$, namely the key at $\mathrm{t}_{2}$, is not able to open door $\mathrm{x}$. So, perfect duplicates do not necessarily share the disposition to open door $x$. The disposition is extrinsic. (McKitrick, 2003: 159) 
In other words, the keys at $t_{1}$ and $t_{2}$ are perfect duplicates, but the door has different locks, so the key at $\mathrm{t}_{1}$ can open the door while the key at $\mathrm{t}_{2}$ cannot. Thus, the former has the disposition to open the door whereas the latter does not. The perfect duplicates do not share the disposition due to the different environments. It follows that the disposition is extrinsic.

It seems to me that McKitrick has contradicted herself. On the one hand, she endorses Shoemaker's contention that the key at $t_{2}$ does not have the disposition to open the door because it does not fit the new lock, i.e., because it is impossible for the disposition to manifest under the current circumstances. On the other hand, she (2003: 157, footnote) endorses David Armstrong's contention that "having the disposition does not entail manifestation of the disposition: a piece of glass may be brittle and yet never break" (Armstrong, 1973: 8). Armstrong would say that the glass is brittle, as long as there is a counterfactual true of the glass. There is such a counterfactual: the glass would break if it were struck by a hammer. By parity of reasoning, he would also say that the key at $t_{2}$ has the disposition to open the door, as long as there is a counterfactual true of the key. There is such a counterfactual: the key would open the door, if the new lock were replaced with the old lock. Thus, Shoemaker's contention is inconsistent with Armstrong's contention, and McKitrick endorses both contentions.

McKitrick claims that "every property is either intrinsic or extrinsic" (2003: 158). It follows that she would characterize the disposition of the key to open the door as only extrinsic. The suggestion that the disposition is extrinsic, however, contradicts Lewis's and McKitrick's definitions of intrinsic and extrinsic properties. The key has the disposition simply because it has a certain shape and solidity. Its shape and solidity are properties that the key has in virtue of the way it is, not in virtue of the relation that it bears to another object. So the disposition is intrinsic. Moreover, the disposition is reducible to the atomic structure of the key, which is a property that the key has in virtue of the way it is, not in virtue of the relation that it bears to another object. Therefore, McKitrick's definition of extrinsic disposition contradicts Lewis's and her definitions of intrinsic and extrinsic properties.

Under McKitrick's framework, an extrinsic disposition cannot be used to explain why an object interacts with another object as it does. After all, she claims that an extrinsic disposition is causally inert:

Can dispositions be causally efficacious? Some philosophers say that a disposition is causally efficacious because it is identical to some intrinsic, first-order property of the disposed object. However, if the disposition in question is extrinsic, that move is not an option. (McKitrick, 2003: 155)

Consider, for example, that the opening of the door is a kind of motion. It is mysterious how the motion of the door can be generated by the causally inert property of the key. The extrinsic disposition cannot make any difference to the way the key interacts with the door. So it is wrong to say that the key opens the door because the key has the extrinsic disposition to open the door.

As we noted at the end of Section 2, according to McKitrick's definition, an extrinsic disposition does not make any intrinsic difference to an object. On her definition, the key remains intrinsically the same, even if the old lock is replaced with the new lock. Thus, it is illegitimate to invoke an extrinsic disposition to explain why an object interacts with another object as it does. An interaction is an event that brings about changes to the interacting objects. Such changes cannot be explained in terms of the objects' extrinsic dispositions because the extrinsic dispositions do not make any intrinsic difference to the interacting 
objects. In sum, the suggestion that the disposition of the key to open the door is extrinsic goes against Ockham's razor in that it posits the existence of an unobservable property despite the absence of any explanatory gain.

How does my definition of disposition handle the example of the key? There is a counterfactual true of the key, viz., if the key is turned, it would interact with the door in a certain manner. The interaction means that the key acquires negligible scratches, and the door opens, which is a salient event. Thus, the example of the key is similar to the example of cyanide, which can produce a conspicuous change to a human being. As we have seen in Section 3.1., the disposition of the cyanide to kill human beings is not extrinsic but intrinsic. Therefore, the disposition of the key to open the door is also not extrinsic but intrinsic.

My definition of disposition is better than McKitrick's for the following two reasons. First, it satisfies Ockham's razor. My definition does not posit the existence of the gratuitous properties, extrinsic dispositions. Yet, under the framework of my definition, we can explain why an object interacts with another object as it does. Second, my definition is consistent with Lewis's and McKitrick's definitions of intrinsic and extrinsic properties. It enshrines their definitions because it is not committed to the existence of extrinsic dispositions.

In addition to the example of the key, McKitrick offers examples of weight and water (2003: 159-161). So far as I can tell, however, they are similar to the example of the key. My responses to these examples can be extrapolated from my response to the example of the key sketched in this section. In the interest of saving space, I skip them and move onto the example of vulnerability.

\subsection{Vulnerability}

McKitrick argues that vulnerability is a disposition in the sense that "something is vulnerable if it is disposed to suffer harm as a result of an attack" (2003: 161). Suppose that a city can be destroyed by bombs dropped from an airplane. Thus, it has the disposition of being vulnerable. Suppose now that a defense system is built outside of the city. Whenever an unfriendly airplane approaches, the defense system activates and shoots the airplane down with $100 \%$ accuracy. The city is no longer vulnerable to an airstrike. Thus, the city is vulnerable or not, depending on whether the defense system is in place, i.e., depending on what goes on outside of the city. The city before and after the defense system is built is perfect duplicates, but they do not share the disposition, vulnerability. It follows that vulnerability is extrinsic.

How should we evaluate McKitrick's contention that the city has the extrinsic disposition of being vulnerable? It is problematic to say that the city loses the disposition of being vulnerable once the defense system is built outside of the city. After all, an object has a disposition, even if the disposition does not manifest, as Armstrong contends. Hence, the city can be vulnerable, even if it does not suffer harm as a result of an attack. In addition, there is a counterfactual true of the city, viz., if the defense system were destroyed, the city would be vulnerable. Therefore, the city retains the disposition of being vulnerable, even if the defense system is built outside of it.

Moreover, vulnerability of the city is an intrinsic property of the city, just as fragility of the glass is an intrinsic property of the glass. There is no relevant difference between the example of the glass and the example of the city. The hammer strikes the glass, and the glass breaks. Analogously, the bombs strike the city, and the city is destroyed. Fragility of the glass is reducible to the atomic structure of the glass. Similarly, vulnerability of the city is reducible to the atomic structure of the city. So both fragility and vulnerability are intrinsic properties. 


\subsection{Visibility and Recognizability}

McKitrick claims that "[v]isibility has a manifestation, being seen, which occurs in the circumstances of manifestation, being looked at by the appropriate type of perceiver" (2003: 162). An astronomer might say that Saturn is visible in the night sky. Saturn's being looked at is the circumstances of manifestation, and Saturn's being seen is a manifestation. The counterfactual is true of Saturn that if Saturn is looked at, it would be seen. The overtly dispositional locution 'the disposition to be seen when looked at' refers to the visibility of Saturn. It follows that Saturn has the disposition to be seen. McKitrick argues that this disposition is extrinsic because Saturn "can cease to be visible without undergoing intrinsic change" (McKitrick, 2003: 162). Saturn would not be visible, for example, if there are thick clouds on the sky blocking the astronomer's view.

What are we to make of this example? First of all, to say that Saturn has the disposition of being visible clashes with what McKitrick says about the concept of disposition in another place in her paper. She says that an object has a disposition just in case it is prone to act in a certain way:

I have outlined a standard philosophical characterization of dispositions. On this understanding, having a disposition is not distinct from being prone to act in a certain way. (McKitrick, 2003: 171)

On her account, then, to say that Saturn has the disposition to be seen is to say that Saturn is prone to act in a certain way, and that to be seen is to act in a certain way. It is, however, a stretch to say that to be seen is to act in a certain way. To act in a certain way means to respond to a stimulus in a certain way. To be seen, however, does not count as a response to a stimulus.

Under my definition of disposition, Saturn does not have the disposition to be seen. Recall that my definition requires that an object should interact with another object, and that the interaction means that the two objects undergo certain changes. When the astronomer sees Saturn, he is not interacting with Saturn. He undergoes a certain change as a result of his looking at Saturn, but Saturn does not undergo any change as a result of his looking at it. Therefore, we cannot attribute the disposition of being visible to Saturn.

We should not let the term 'visibility' mislead us. The morphological structure of 'visibility' is analogous to that of 'fragility' in that both words end with the suffix, 'ity.' Since 'fragility' refers to a disposition of an object, one might be tempted to think that 'visibility' also refers to a disposition of an object. On close examination, however, 'visibility' does not. It is wrong for metaphysicians to rely on the structure and use of language to investigate reality. It is a mistake, for example, to rely on the uses of the terms 'the Earth' and 'the Sun' to investigate the relationship between the Earth and the Sun. After all, we still say, "The Sun rises in the east and sets in the west," when in fact the Earth turns eastward on its axis. Metaphysicians should not be misled by how language is used.

When an astronomer says, "Saturn is visible," the grammatical structure of the sentence gives the impression that he attributes the disposition of being visible to Saturn. In reality, however, he does not. He could say, "I see Saturn" instead of saying "Saturn is visible." But he says, "Saturn is visible" because 'I see Saturn' is merely a report of his subjective experience, whereas 'Saturn is visible' appears to be a report of an objective state of affairs. The assertion that Saturn is visible is, but the assertion that he sees Saturn is not, loaded with the counterfactual that if other people are in similar perceptual circumstances, they too would see it. By saying "Saturn is visible," the astronomer is implying that other people would see it as he does. He is not implying that Saturn has the disposition to be seen. If we interpret his 
locution 'Saturn is visible' as ascribing the disposition of being visible to Saturn, we have no choice but to ascribe falsity to his belief because being seen is not a disposition and therefore Saturn cannot have it.

McKitrick (2003: 163) also argues that recognizability is an extrinsic disposition, providing the example of Bill Clinton. As far as I can tell, however, recognizability is no different in kind from visibility. My response to her discussion of recognizability can be inferred from my response to her discussion of visibility sketched in this section. In the interest of saving space, I skip her discussion of recognizability.

\section{Lonely Universe}

Imagine that there is nothing but a piece of glass in the universe. Does the glass have the disposition of being fragile in this lonely universe? Our intuition says yes because the glass in this possible world is intrinsically no different from a piece of glass in the actual world (Christian Wüthrich, Personal Communication). If the latter is fragile, so is the former. This reasoning goes hand in hand with Armstrong's contention that "having the disposition does not entail manifestation of the disposition: a piece of glass may be brittle and yet never break (Armstrong, 1973: 8)."

Under the framework of McKitrick's definition of disposition, however, we cannot attribute fragility to the glass in the lonely universe. Recall that her definition holds that an object has a disposition if and only if there are a manifestation, the circumstances of manifestation, a counterfactual true of the object, and an overtly dispositional locution referring to the disposition. In the lonely universe, there are no circumstances under which an object can exhibit a manifestation. Hence, there is neither a manifestation nor the circumstances of the manifestation. There is no adequate ground for attributing the disposition to the glass.

In contrast, under the framework of my definition of disposition, we can attribute fragility to the glass in the lonely universe. Recall that my definition holds that an object has a disposition if and only if there is a counterfactual true of the object that under a certain condition, it would interact with another object in a certain manner. In the lonely universe, the counterfactual is true of the glass that if the glass were struck by a hammer, it would break. Since we have the true counterfactual, we can attribute fragility to the glass.

One might raise the following objection. McKitrick states that the "manifestation need not occur for something to possess the disposition" (2003: 157). She also tells us that in order to determine whether a property is a disposition or not, we should ask, "Is there a certain counterfactual that is typically true of things that possess P?" (McKitrick, 2003: 158). There is a counterfactual true of the glass in the lonely universe, viz., if the glass were struck by a hammer, it would break. So under McKitrick's framework, we can attribute fragility to the glass in the lonely universe.

It seems to me, however, that McKitrick contradicted herself. As cited in Section 3.2., she also tells us that we should ask the following two questions to determine whether a property is a disposition or not:

Does $\mathrm{P}$ have a characteristic manifestation? Are there circumstances that would typically bring about the occurrence of this manifestation? (McKitrick, 2003: 158)

In my view, we should not ask these two questions, if manifestation need not occur for something to possess a disposition. Thus, McKitrick faces the task of reconciling the following two claims. (1) Manifestation need not occur for something to possess a disposition. (2) We should ask whether a property has a characteristic manifestation and whether there are 
circumstances that would typically bring about the occurrence of this manifestation to determine whether the property is a disposition or not.

\section{Conclusion}

McKitrick's definition and six examples of extrinsic dispositions lie at the heart of her case for the existence of extrinsic definitions. Her case stands or falls, depending on whether the definition and the examples are tenable. I offered an alternative definition of disposition. The key difference between them is that my definition requires, whereas her definition does not, that under a certain condition, an object to which we ascribe a disposition should interact with another object in a certain manner. This requirement of counterfactual interaction sets the two definitions apart from each other.

McKitrick's examples of extrinsic dispositions are problematic. Concerning her example of the key, I argued that her position goes against Ockham's razor and contradicts Lewis's and her definitions of intrinsic and extrinsic properties. Concerning the example of the city, I argued that vulnerability of the city is an intrinsic property of the city, just as fragility of the glass is an intrinsic property of the glass. Concerning the example of Saturn's visibility, I argued that we cannot attribute the disposition of being visible to Saturn because it is not clear that to be seen counts as a response to a stimulus, and because there is no interaction between the astronomer and Saturn.

My definition of disposition is better than McKitrick's definition of disposition for the following three reasons: First, the resulting ontology of my definition is slimmer than that of her definition. Second, her definition contradicts, but mine does not, Lewis's and her definitions of intrinsic and extrinsic properties. Third, my definition can, but her definition cannot, accommodate our intuition that the glass in the lonely universe is fragile. In short, McKitrick's case for extrinsic dispositions fails.

\section{References}

Armstrong, David (1973). Belief, Truth, and Knowledge. New York: Cambridge University Press.

Johnston, Mark (1992). "How to Speak of the Colors", Philosophical Studies 68 (3): 221-263.

Lewis, Davis (1986). On the Plurality of Worlds. Blackwell Publishing.

$$
\text { (1997). “Finkish Dispositions”, Philosophical Quarterly } 47 \text { (187): 143-158. }
$$

McKitrick, Jennifer (2003). “A Case for Extrinsic Dispositions”, Australasian Journal of Philosophy 81 (2): 155-174.

Molnar, George (1999). “Are Dispositions Reducible?” Philosophical Quarterly 50 (194): 117.

Shoemaker, Sydney (1984). Identity, Cause, and Mind. Cambridge: Cambridge University Press. 